\title{
Simulation of Propagation of Action Potentials in Cardiac Tissue with an Inhomogeneous Distribution of Extracellular Potassium
}

\section{Sudarshan Dhungana ${ }^{1}$ and Piyush Kar ${ }^{2 *}$}

${ }^{1}$ Oakland University, Rochester, MI, USA

${ }^{2}$ University of Alberta, Edmonton, Alberta, Canada

\begin{abstract}
We report simulation of experimental data for heterogeneous distribution of extracellular potassium, by developing and applying a mathematical model formulated by incorporating a Luo-Rudy representation of the cell membrane current into the bidomain model of the cardiac tissue. Our model serves to examine propagation of extra cellular potassium from a region of low potassium (normal) to a region of high potassium (ischemic). Starting with computer simulation of unipolar stimulation of cardiac tissue, we reproduced results of the passive bidomain model, and also simulated the space-clamped Luo Rudy model. Finally, we combined the Luo-Rudy model with the bidomain model. The diastolic threshold for anodal stimulation first decreased and then increased with increasing potassium concentration $\left([K]_{0}\right)$, reaching a minimum value at $[K]_{0}=12 \mathrm{mM}$.
\end{abstract}

Keywords: Mathematical modeling; Luo-Rudy model; Bidomain model; Extracellular potassium; Cardiac tissue; Action potential

\section{Introduction}

Our mind works and our heart beats as long as there is propagation of action potentials in our body. Action potentials are generated by the inward and outward movements of numerous ions, such as $\mathrm{Na}^{+}$, $\mathrm{K}^{+}, \mathrm{Ca}^{2+}, \mathrm{Cl}^{-}$across the cell membrane. These action potentials travel in the form of waves through the various chambers of the heart. To fully understand the heart, a detailed knowledge of action potential propagation is essential.

In unhealthy (ischemic) tissue, the extracellular potassium concentration, $[K]_{0}$, is elevated. High $[K]_{0}$ is believed to predispose cardiac tissue to break excitation (Roth and Patel) [1], which in turn plays an important role in re-entry induction and defibrillation. Because fibrillation raises extracellular $[K]_{0}$ levels, break excitation may play a more important role in defibrillation than is suggested by simulations and experiments using normal $[K]_{0}$ values. In their 2003 paper, Roth and Patel theoretically simulated action potential data in a $1 \mathrm{~cm}^{2}$ tissue verifying that the action potential may fail to propagate in an ischemic region. The wave propagates for $[K]_{0}=2$ to $12.5 \mathrm{mM}$ and at higher potassium concentrations the wave dies out.

Sidorov et al. studied propagation in ischemic tissue experimentally. Their optical mapping experiment in a rabbit heart using $[K]_{0}$ values of $4,6,8,10$ and $12 \mathrm{mM}$ indicated that spatial heterogeneity of the action potential duration (APD) restitution, created with regional elevation of $\left[\mathrm{K}^{+}\right]_{0}$, can lead to action potential (AP) instability, 2:1 block, and reentry induction [2].

The goal of this research is to simulate Sidorov et al. experiment using a mathematical model by incorporating a Luo-Rudy representation of the cell membrane current with the bidomain model of the cardiac tissue, and to examine propagation of potassium from a normal to an ischemic region.

\section{Methods}

\section{Luo rudy model}

Hodgkin and Huxley published a landmark paper that paved the way for subsequent electrophysiological models. They successfully explained the propagation of action potentials in the giant squid axon by representing the membrane resistance as resulting from ion flow through three ion channels: sodium, potassium and leakage [3]. Each pathway was accompanied by a battery that had a potential equal to the Nernst potential for that ion. Variable resistances signifying the various ion channels opening and closing were expressed in terms of their conductance. The probability of opening or closing of these ionic channels was governed by three gating parameters $m, n$ and $h$ following the first order kinematics.

$$
\frac{d s_{i}}{d t}=\dot{a}_{i}\left(1-s_{i}\right)-\beta_{i} s_{i}
$$

In eq. (1), $\alpha_{i}$ and $\beta_{i}$ represent the rate of opening and closing of the $m, n$, and $h$ gates. These rate constants depended on the transmembrane voltage.

Hodgkin and Huxley wrote down the rate of change of membrane potential as

$$
\frac{d V}{d t}=-\left(\frac{1}{C}\right)\left(I_{i}+I_{\text {stim }}\right)
$$

Following the mathematical formulation of Hodgkin and Huxley, in 1991 Luo and Rudy developed a model of the ventricular cardiac action potential. They introduced and analyzed more ion channels then other contemporary physiological models. They replaced the three ionic current terms in Hodgkin and Huxley's model with six other currents. They were a fast inward sodium $\mathrm{Na}^{+}$current $I_{\mathrm{Na}}$, a slow inward calcium $\mathrm{Ca}^{++}$current $I_{S i}$, time dependent delayed rectifier potassium $\mathrm{K}^{+}$current $I_{k}$, and time-independent potassium inwardly rectifying current $I_{k l}$, the plateau $\mathrm{K}^{+}$current $I_{k p}$, and a time independent background current $I_{b}$ The membrane current depended on $\left[\mathrm{K}^{+}\right]_{0}$ as a parameter in this model. Considering a membrane patch of area $1 \mathrm{~cm}^{2}$ with capacitance $1 \mu \mathrm{F} / \mathrm{cm}^{2}$ equation (2) can be rewritten as

*Corresponding author: Piyush Kar, University of Alberta, Edmonton, Alberta, Canada, Tel: 514-7122130; E-mail: pkar1@ualberta.ca

Received November 04, 2015; Accepted November 25, 2015; Published November 30, 2015

Citation: Dhungana S, Kar P (2015) Simulation of Propagation of Action Potentials in Cardiac Tissue with an Inhomogeneous Distribution of Extracellular Potassium. J Comput Sci Syst Biol 8:6 373-379. doi:10.4172/jcsb.1000212

Copyright: (C) 2015 Dhungana S, et al. This is an open-access article distributed under the terms of the Creative Commons Attribution License, which permits unrestricted use, distribution, and reproduction in any medium, provided the original author and source are credited. 


$$
\frac{d V}{d t}=-\left(\frac{1}{C}\right)\left(I_{N a}+I_{s i}+I_{k}+I_{k 1}+I_{k p}+I_{b}+I_{s t i m}\right)
$$

All these ionic currents also depend on the gating variables, which are actually probabilistic values indicating the closing and opening of various ion channels. The time variation is given as

$$
\frac{d y}{d t}=\frac{\left(y_{\infty}-y\right)}{\tau_{y}}
$$

where,

$$
\tau_{y}=\frac{1}{\left(\alpha_{y}+\beta_{y}\right)}
$$

and,

$$
y_{\infty}=\frac{\alpha_{y}}{\left(\alpha_{y}+\beta_{y}\right)}
$$

Here, $y$ can be any of the gating variables $(m, h, \ldots), \tau_{y}$ is the time constant, and $\mathrm{y}_{\infty}$ is the steady state value of $y . \alpha_{y}$ and $\beta_{y}^{y}$ are voltage dependent rate constants.

The fast inward sodium current in eq. (3) was described using the Ebihara-Johnson model [4] (Ebihara and Johnson) coupled with the slow inactivation gate ( $\mathrm{j}$ ) of the Beeler-Reuter model (Beeler and Reuter) [5],

$$
I_{N a}=\bar{G}_{N a} m^{3} h j\left(V-E_{N a}\right)
$$

where, $\bar{G}_{N a}$ is the ,...maximum conductance of the sodium channel (23 $\left.\mathrm{mS} / \mathrm{cm}^{2}\right) ; \mathrm{m}$ the activation parameter and $\mathrm{h}$ the inactivation parameter; and $E_{\mathrm{Na}}$ is the reversal potential of sodium.

$$
\left[E_{N a}=\left(\frac{R T}{F}\right) \ln \left(\frac{[N a]_{0}}{[N a\}_{i}}\right)\right], E_{N a}=54.4 m V
$$

The formulation of $I_{S i}$ comes from the Beeler-Reuter model

$$
E_{s i}=7.7-13.0287 \ln \left([\mathrm{Ca}]_{i}\right)
$$

where,

$$
E_{s i}=7.7-13.0287 \ln \left([\mathrm{Ca}]_{i}\right)
$$

and, $d$ and $f$ are two other gating parameters.

The delayed outward rectifier current $I_{k}$ was based on a formulation proposed by Shibasaki and included a time-dependent activation gate $\mathrm{X}$, a time independent (fast) inactivation gate $\mathrm{X}_{\mathrm{i}}$ capturing the inward rectification properties of the delayed rectifier current, and a conductance modulated by extracellular $\mathrm{K}^{+}$concentration [6].

$$
E_{K}=-77 \mathrm{mV}, \overline{G_{K}}=0.282 \sqrt{\frac{[K]_{0}}{5.4}}
$$

$$
E_{K}=-77 \mathrm{mV}, \overline{G_{K}}=0.282 \sqrt{\frac{[K]_{0}}{5.4}}
$$

The formulation of $I_{k 1}$ was based on patch clamp data of Sakmann and Trube as well as other data from single ventricular myocytes [7].

$$
I_{K 1}=\overline{G_{K 1}} K 1_{x}\left(V-E_{K 1}\right)
$$

where,

$$
\overline{G_{K 1}}=0.6047 \sqrt{\frac{[K]_{0}}{5.4}}
$$

The voltage-dependent and time independent plateau current that activates at depolarized plateau potentials was identified by Yue and Marban [8],

$$
I_{K \rho}=0.0183 K_{\rho}\left(V-E_{K \rho}\right)
$$

Where, $E_{k p}=E_{k 1}$

$$
K_{p}=\frac{1 .}{\left\{1+\exp \left[\frac{7.488-V}{5.98}\right]\right\}}
$$

The background current is formulated as

$$
I_{b}=\overline{G_{b}}\left(V-E_{b}\right)
$$

where, $E_{b}=-59.87 \mathrm{mV}, E_{b}=-59.87 \mathrm{mV}$

Then the total time independent current is

$$
I_{K 1(T)}=I_{K 1}+I_{K \rho}+I_{b}
$$

Finally the rate of calcium uptake is given by

$$
\frac{d\left([\mathrm{Ca}]_{i}\right)}{d t}=-10^{-4} \cdot I_{s i}+0.07\left(10^{-4}-[\mathrm{Ca}]_{i}\right)
$$

The complete listing of all the governing equations, parameters and their corresponding values can be found in Appendix.

\section{Bidomain model}

The two or three dimensional cable model, more commonly known as the bidomain model, describes the interaction of electric current with cardiac tissue. It was developed in the late 1970's (Muler and Markin, Miller and Geselowitz, Tung) [9-11] and was applied to more problems in cardiac electrophysiology in the 1980s (Plonsey and Barr, Barr and Plonsey, Roth and Wikswo) [12-14].

Here we will solve the coupled parabolic and elliptic equations using a finite difference technique (Roth) and discuss some consequences of unipolar stimulation of cardiac tissue [15]. The first analysis of this kind of work was done by Sepulveda et al. [16]. The starting point here is to solve the set of two coupled partial differential equations governing the transmembrane potential, $V_{m}$, and extracellular potential, $V_{e}$

$$
\begin{aligned}
& C_{m} \frac{\partial V_{m}}{\partial \hat{a}}=-J_{i o n}-\frac{1}{x}\left[g_{e x} \frac{\partial^{2} V_{e}}{\partial \partial^{2}}+g_{e y} \frac{\partial^{2} V_{e}}{\partial^{2}}\right] \\
& \left(g_{i x}+g_{e x}\right) \frac{\partial^{2} V_{e}}{\partial x^{2}}+\left(g_{i y}+g_{e y}\right) \frac{\partial^{2} V_{e}}{\partial y^{2}}=-g_{i x} \frac{\partial^{2} V_{m}}{\partial x^{2}}-g_{i y} \frac{\partial^{2} V_{m}}{\partial y^{2}}
\end{aligned}
$$

To solve the parabolic type of equation, we use the simple Euler method. The idea here is to solve eq. (20) for the new value of $V_{m}$, and then solve the elliptic eq. (21) for the new value of $V_{e}$ using the new value for $V_{m}$ as a source term, by over-relaxation.

By, rearranging, we get,

$$
\begin{aligned}
& \left(g_{i x}+g_{e x}\right)\left[\frac{V_{e}(i+1, j)-2 V_{e}(i, j)+V_{e}(i-1, j)}{\Delta x^{2}}\right]+\left(g_{i j}+g_{e j}\right)\left[\frac{V_{e}(i, j+1)-2 V_{e}(i, j)+V_{e}(i, j-1)}{\Delta y^{2}}\right]= \\
& -\frac{g_{i x}}{\Delta x^{2}}\left[V_{m}(i+1, j)-2 V_{m}(i, j)+V_{m}(i-1, j)\right]-\frac{g_{i y}}{\Delta y^{2}}\left[V_{m}(i, j+1)-2 V_{m}(i, j)+V_{m}(i, j-1)\right]
\end{aligned}
$$

In eq. (22), let,

$$
\begin{aligned}
& X_{1}=-2\left(\frac{g_{i x}+g_{e x}}{\Delta x^{2}}+\frac{g_{i y}+g_{e y}}{\Delta y^{2}}\right) \\
& X_{2}=-\left(\frac{g_{i x}+g_{e x}}{\Delta x^{2}}\right) \\
& X_{3}=-\left(\frac{g_{i y}+g_{e y}}{\Delta y^{2}}\right) \\
& X_{5}=-\frac{g_{i y}}{\Delta y^{2}} \\
& X_{5}=-\frac{g_{i y}}{\Delta y^{2}}
\end{aligned}
$$


Citation: Dhungana S, Kar P (2015) Simulation of Propagation of Action Potentials in Cardiac Tissue with an Inhomogeneous Distribution of Extracellular Potassium. J Comput Sci Syst Biol 8:6 373-379. doi:10.4172/jcsb.1000212

So, we can write an expression for extracellular potential, $V_{e}$ in terms of $V_{m}$, as follows:

$$
\begin{aligned}
& V_{e}(i, j)=\left(\frac{X_{2}}{X_{1}}\right)\left(V_{e}(i-1, j)+V_{e}(i+1, j)\right) \\
& +\left(\frac{X_{3}}{X_{1}}\right)\left(V_{e}(i, j-1)+V_{e}(i, j+1)\right) \\
& +\left(\frac{X_{4}}{X_{1}}\right)\left(V_{m}(i+1, j)-2 V_{m}(i, j)+V_{m}(i-1, j)\right)
\end{aligned}
$$

\section{Results and Discussion}

By developing a code using the FORTRAN software, we simulated the distribution of extracellular potassium by using the bidomain model with a LR action potential. Sepulveda et al. used a passive bidomain model to study unipolar stimulation of cardiac tissue [16]. They found that near a unipolar cathode, the tissue is strongly depolarized under the cathode, and more weakly hyperpolarized in two regions near the cathode along the fiber direction. Figure 1 shows our model generated transmembrane potential produced around the cathode, located at the origin.

In presence of the cathode the tissue is depolarized (positive transmembrane potential) in a dogbone shape. However, about 2 $\mathrm{mm}$ from the cathode, in the direction along the fibers, is a region of hyperpolarization (negative), called a "virtual anode". In Figure 1, only one quarter of the tissue is shown, so two virtual anodes exist one on each side of the cathode. The values in the plots are given in volts. Figure 2 shows anodic stimulation, which gives rise to two virtual cathodes on either side of the origin. Figure 3 shows the calculated transmembrane potential during unipolar cathodal stimulation of an anisotropic twodimensional sheet of passive cardiac tissue in a color plot.

A space clamped calculation of the LR action potential was performed in Luo and Rudy's original paper (Luo and Rudy) [17]. Figure 4 shows simulated action potentials for different extracellular concentrations of potassium.

As $[K]_{0}$ decreases, action potential duration increases and resting potential becomes more negative. Figure 5 shows the time course of $I_{\mathrm{K} 1(\mathrm{~T})}, I_{K}$, and $I_{s i}$ during the action potential. A peak in $I_{\mathrm{K} 1(\mathrm{~T})}$ is observed during repolarization. For $[K]_{0}=7 \mathrm{mM}$ the peak is almost $3 \mu \mathrm{A} / \mathrm{cm}^{2}$ but for $[K]_{0}=3 \mathrm{mM}$ it is less than $2 \mu \mathrm{A} / \mathrm{cm}^{2}$. This large difference in $I_{\mathrm{K} 1(\mathrm{~T})}$

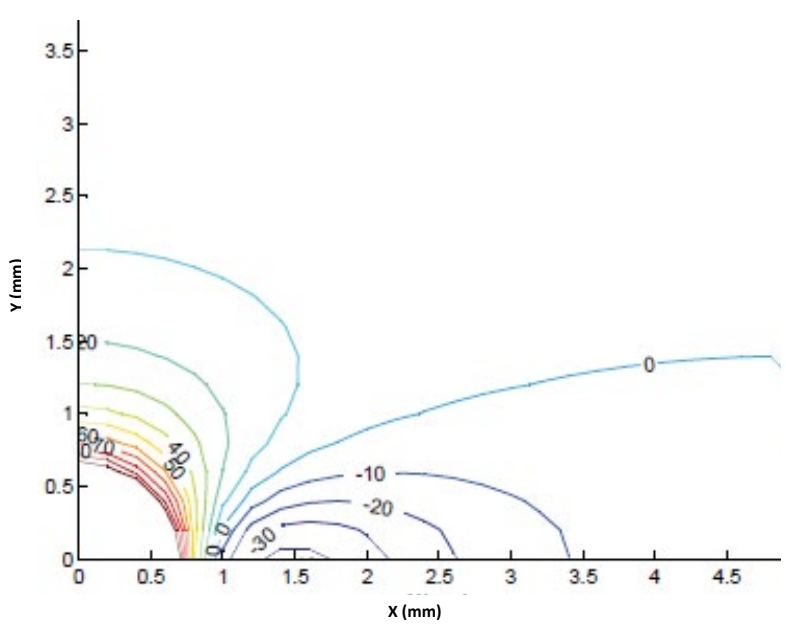

Figure 1: Transmembrane potential induced in a $2 \mathrm{D}$ sheet of tissue by unipolar extracellular cathode, located at the origin. The fibers are along the $\mathrm{X}$ axis.

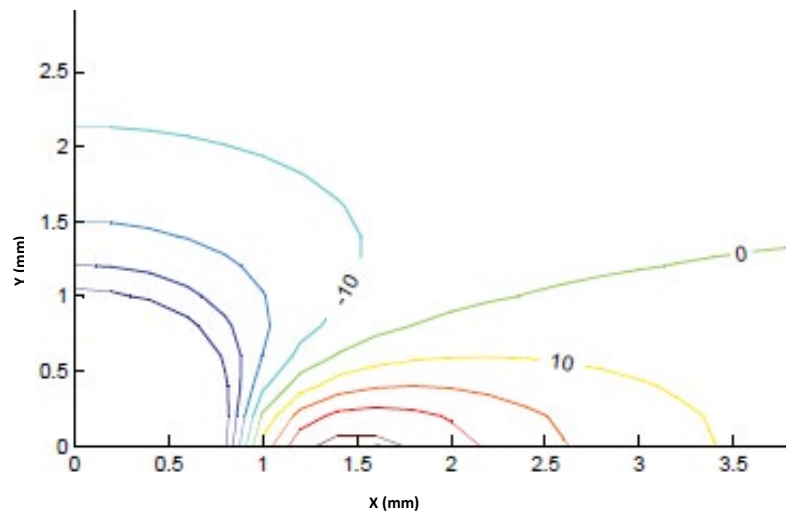

Figure 2: Transmembrane potential induced in a 2D sheet of tissue by unipolar extracellular anode located at the origin.

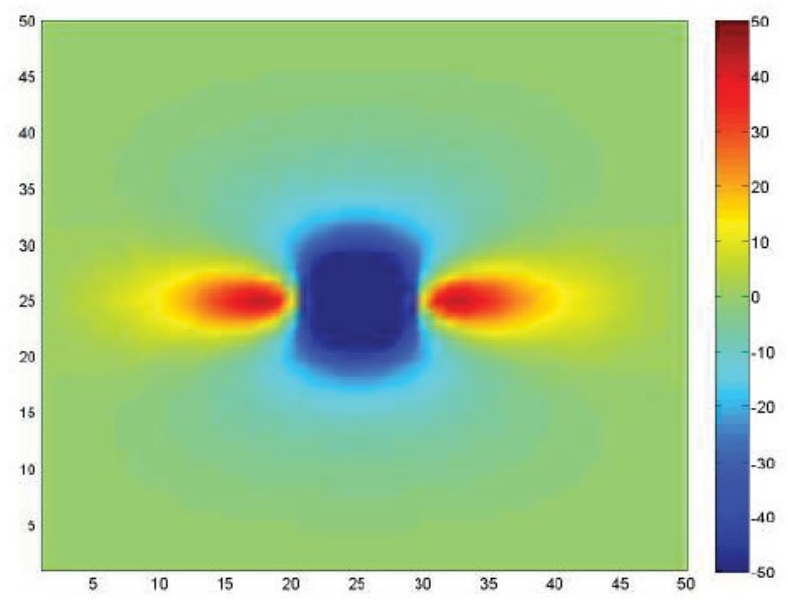

Figure 3: Color plot of cathodal stimulation in a two dimensional sheet of cardiac tissue. The electrode is at the center of the tissue, and the fibers are horizontal.

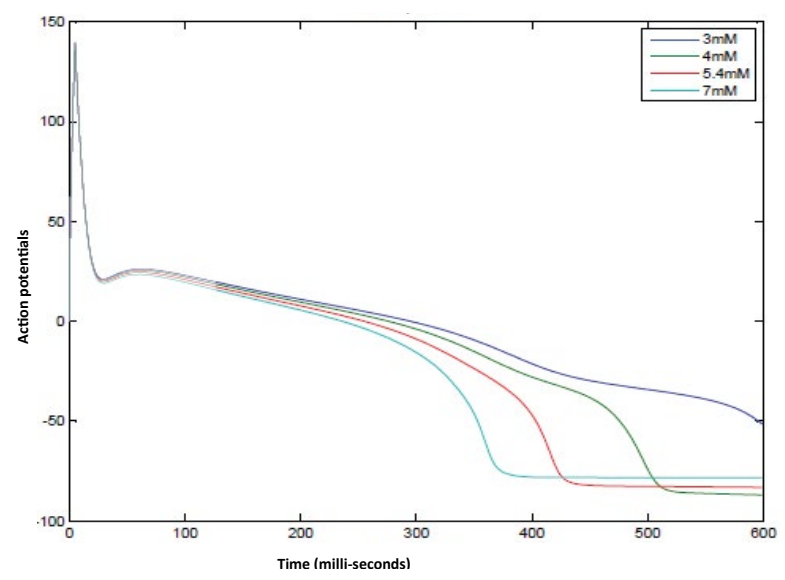

Figure 4: Action potentials for $[K]_{0}=7,5.4,4$, and $3 \mathrm{mM}$, calculated using the space-clamped Luo-Rudy model.

brings about a faster rate of repolarization at higher $[K]_{0}$. Also the peak of $I_{\mathrm{K} 1(\mathrm{~T})}$ occurs earlier (at more positive membrane potential), reflecting an increase of the reversal potential $E_{\mathrm{K} 1}$.

Furthermore, Figures 5-7 indicates that during repolarization the 
Citation: Dhungana S, Kar P (2015) Simulation of Propagation of Action Potentials in Cardiac Tissue with an Inhomogeneous Distribution of Extracellular Potassium. J Comput Sci Syst Biol 8:6 373-379. doi:10.4172/jcsb.1000212

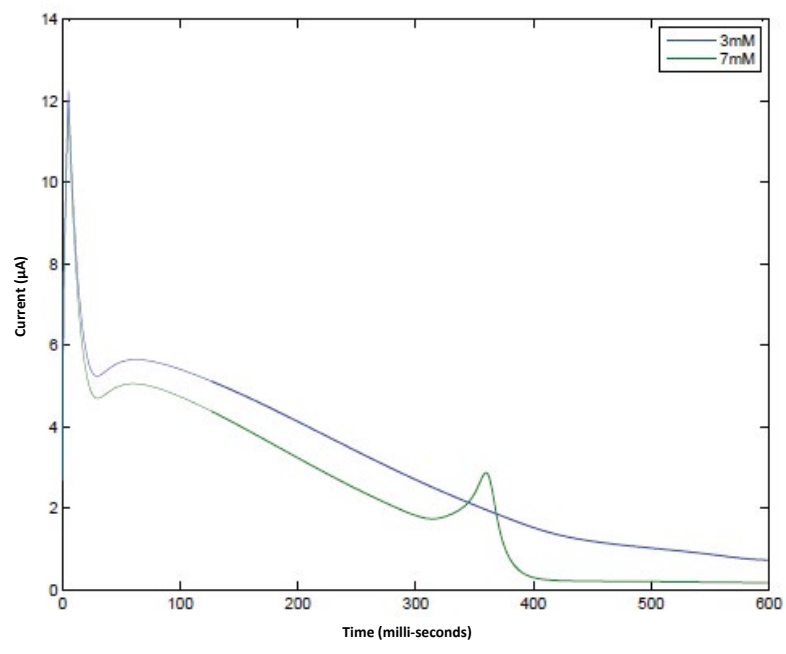

Figure 5: Dependence of ionic current $\mathrm{I}_{\mathrm{K} 1(\mathrm{~T})}$ on $[K]_{0}$.

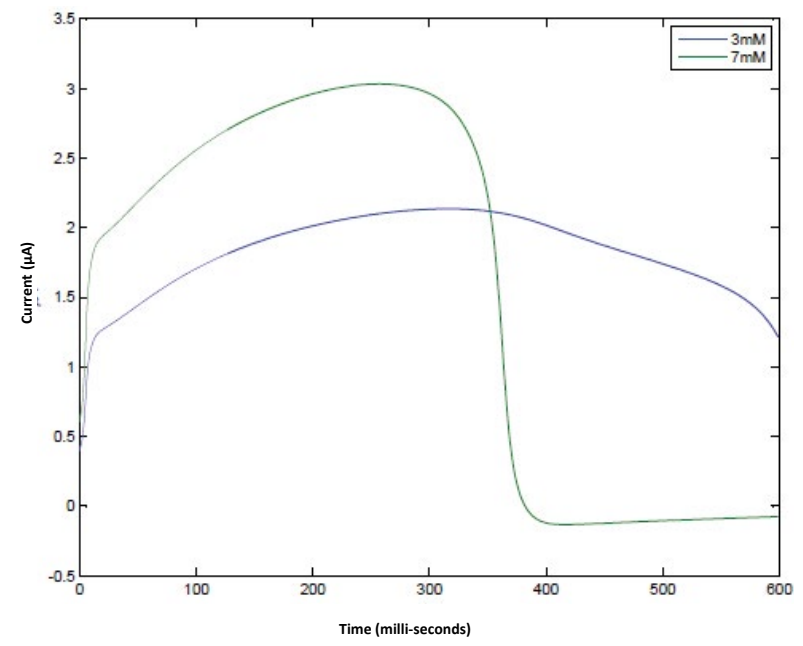

Figure 6: Dependence of $I_{\mathrm{k}}$ on $[K]_{0}$.

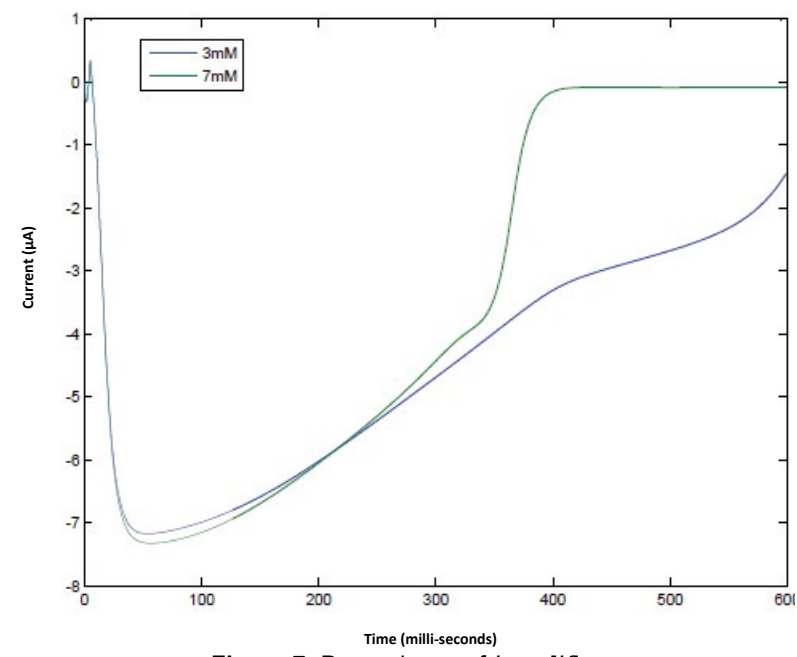

Figure 7: Dependence of $I_{\mathrm{si}}$ on $[K]$. total time independent potassium current depends strongly on $[K]_{0}$, while the time-dependent potassium current $\left(I_{\mathrm{K}}\right)$ and slow inward current exhibit only weak dependence on $[K]_{0}$.

The 1991 Luo-Rudy Phase I model did not describe intracellular $\mathrm{Ca}^{2+}$ cycling, sarcolemmal $\mathrm{Na}^{+}-\mathrm{Ca}^{2+}$ exchange, $\mathrm{Na}^{+}-\mathrm{K}^{+}$pumping and temporal variation of intracellular ion concentrations [17]. Luo and Rudy produced another model called Luo-Rudy phase II model, which is one of the most popular cardiac ventricular cell models even today (Luo and Rudy) [18]. However, because focus is made on extracellular potassium concentration and not on intracellular calcium dynamics, it is not relevant here.

Our next analysis is made by combining the bidomain model with an active Luo-Rudy membrane, which bears similarity with simulations performed previously by Roth [15]. Figure 8 shows stimulation threshold as a function of $[K]_{0}$. The threshold initially decreases as $[K]_{0}$ increases, but then rises abruptly for $[K]_{0}$ above $12 \mathrm{mM}$. Figure 9 shows the resting potential as a function of potassium ion concentration. The resting potential follows approximately the potassium Nernst potential, with a very negative value at low $[K]_{0}$ and then rising to less negative values as $[K]_{0}$ increases.

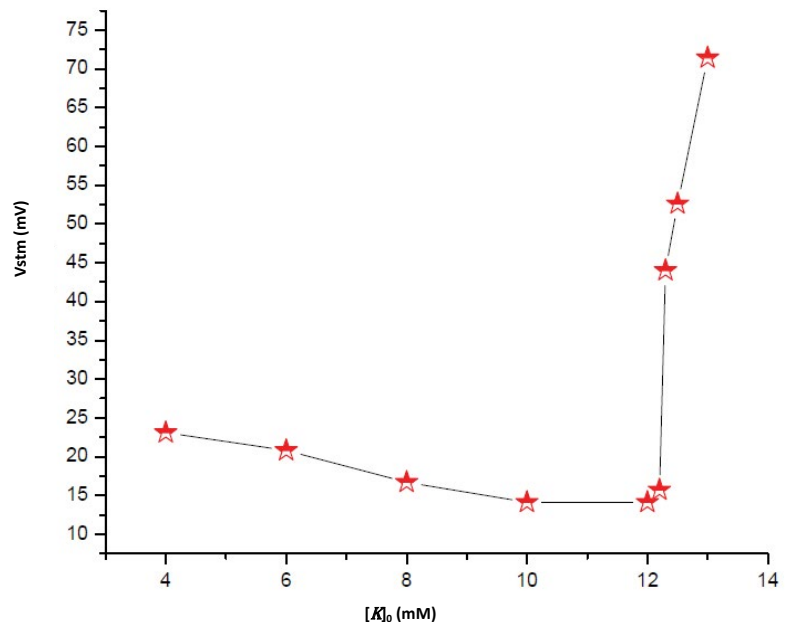

Figure 8: Stimulus threshold voltage as a function of $[K]_{0}$.

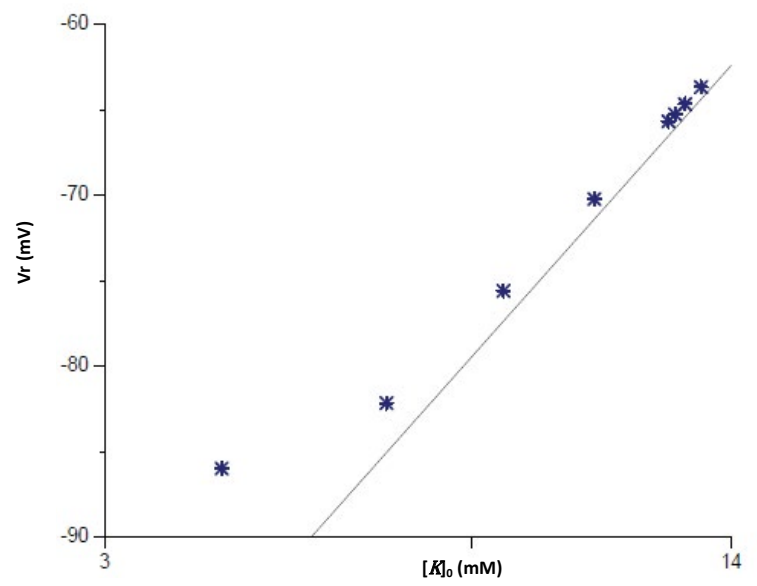

Figure 9: Resting potential as a function of extracellular potassium ion concentration. The straight line represents the potassium Nernst potential. 
Citation: Dhungana S, Kar P (2015) Simulation of Propagation of Action Potentials in Cardiac Tissue with an Inhomogeneous Distribution of Extracellular Potassium. J Comput Sci Syst Biol 8:6 373-379. doi:10.4172/jcsb.1000212

Having verified that the model gives results similar to previous calculations, we can now apply the model to simulating the experiments of Sidorov et al. [2]. We can consider a tissue that has $[K]_{0}=4 \mathrm{mM}$ (normal) on the left half of the tissue, and $[K]_{0}=10 \mathrm{mM}$ (ischemic) on the right (Figure 10). The tissue sheet is $10 \mathrm{~mm}$ by $10 \mathrm{~mm}(100 \times 100$ nodes), with a space step in each direction of $0.1 \mathrm{~mm}$.

We can stimulate the tissue by raising the extracellular potential along the left edge to $20 \mathrm{mV}$ at time $\mathrm{t}=0$. An action potential then propagates across the tissue from left to right. Plots of the transmembrane potential versus time at three locations are shown in Figures 11 and 12. Point $A(x=-4.92 \mathrm{~mm}, y=-4.92 \mathrm{~mm})$ is near the left edge of the tissue sheet in the region of normal $[K]_{0}$, point $\mathrm{B}(\mathrm{x}=-0.025$, $y=-0.025$ ) is at the center (just on the boundary between normal and ischemic tissue), and point $\mathrm{C}(\mathrm{x}=4.9 \mathrm{~mm}, \mathrm{y}=4.9 \mathrm{~mm})$ is near the right edge in the region of elevated $[K]_{0}$.

The second stimulus was applied at various times, but never found

\begin{tabular}{|c|c|}
\hline $\mathrm{T}$ & Stimulus \\
\hline $0<t \leq 1$ & $20 \mathrm{Mv}$ \\
\hline $542<t \leq 543$ & $20 \mathrm{mV}$ \\
\hline
\end{tabular}

1 50

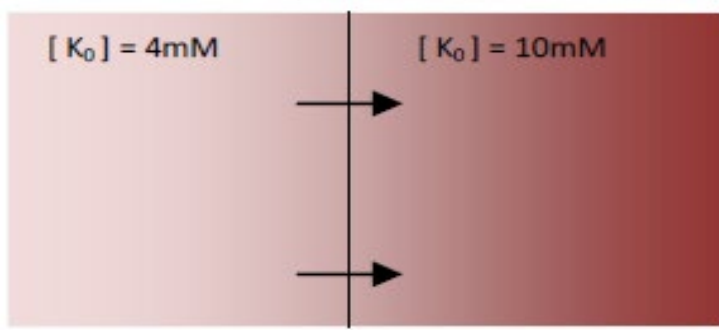

A(-4.9,-4.9)

$\mathrm{B}(-.025,-.025)$

$\mathrm{C}(4.9,4.9)$

Figure 10: Two-dimensional sheet of tissue with normal (left) and ischemic (right) regions. Following an extracellular stimulus of $20 \mathrm{mV}$ along the left edge, applied for the time periods, shown in the table, an action AP wave spreads from normal to ischemic tissue.

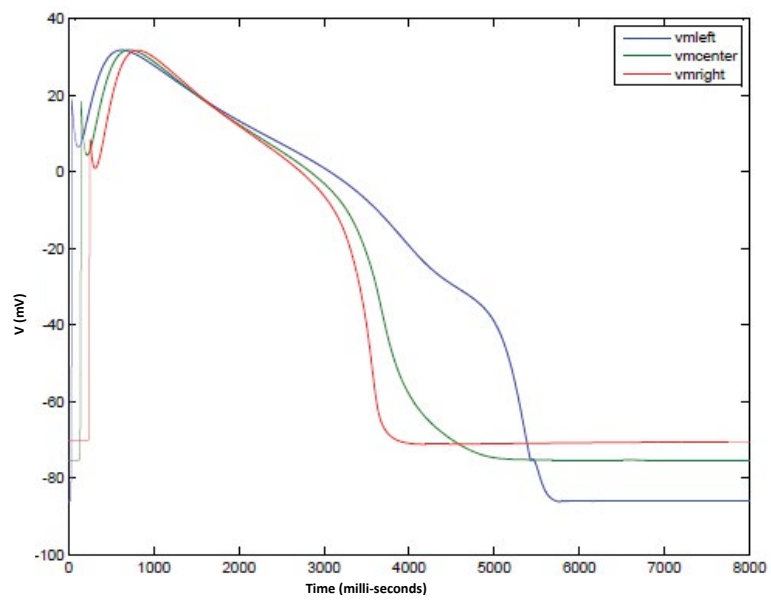

Figure 11: Action potential wave. The $2^{\text {nd }}$ stimulus produced no AP because the tissue is refractory. Time interval chosen to apply stimulus is below the diagram (small table).

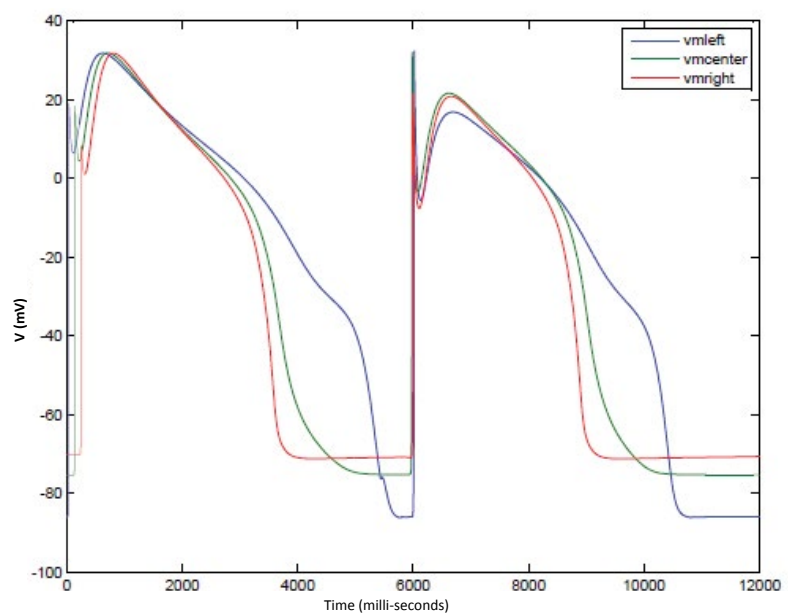

Figure 12: The transmembrane potential when the second stimulus is applied in the interval $542<t \leq 543 \mathrm{~ms}$. It can be observed that both, the first and second action potential waves, pass through the normal and ischemic tissue.

a case where propagation was successful in the normal tissue but then failed to enter the ischemic tissue. Also attempt was made by applying more than two stimuli (up to five) with various timings, and varied the distribution of extracellular potassium so that in one case the left was $5.4 \mathrm{mM}$ and the right was $8 \mathrm{mM}$ (Figure 13), and in another case the left was $8 \mathrm{mM}$ and the right was $12 \mathrm{mM}$ (Figure 14). Nevertheless, after extensive simulations none of these simulations resulted in propagation on the left but then failure on the right.

We validated our calculations by combining both models by verifying previous calculations, in that we reproduced results reported by Sepulveda et al. [16], Luo-Rudy action potential (Luo and Rudy, 1991) and by Roth and Chen $[17,19]$. This exercise brought about the conclusion that resting potential rises with $[K]_{0}$, which tends to make propagation easier (lower threshold) initially as $[K]_{0}$ increases, but makes propagation harder (higher threshold) for very high $[K]_{0}$ (Figures 8 and 9).

Finally the Sidorov et al. experiment was simulated. In their case, the wave front propagated from a region of $[K]_{0}=4 \mathrm{mM}$ to a region of $[K]_{0}=10 \mathrm{mM}$ in a Langendorff-perfused rabbit heart [2]. They often found cases in which propagation was successful in the normal $(4 \mathrm{mM})$ tissue but then failed at the boundary between the normal and ischemic $(10 \mathrm{mM})$ tissue. Here this behavior was never observed. The reason appears to be the dependence of action potential duration on $[K]_{0}$ In the Luo-Rudy model, the action potential duration decreases as $[K]_{0}$ increases, so that the refractory period in the ischemic region is short. Any action potential that can propagate through the normal tissue (with a long refractory period) should have no problem also propagating through the ischemic tissue (with a short refractory period). Interestingly, in Sidorov et al. data, the action potential duration (and therefore the refractory period) appeared insensitive to extracellular potassium ion concentration. This appears to be the primary difference between our simulations (reported herein) and their experiments. Future simulations of Sidorov et al.'s data would need to include a model of the membrane kinetics that properly captures the $[K]_{0}$ dependence of the action potential duration.

\section{Conclusions}

We simulated Sidorov's experiment, in rabit heart, using 
Citation: Dhungana S, Kar P (2015) Simulation of Propagation of Action Potentials in Cardiac Tissue with an Inhomogeneous Distribution of Extracellular Potassium. J Comput Sci Syst Biol 8:6 373-379. doi:10.4172/jcsb.1000212

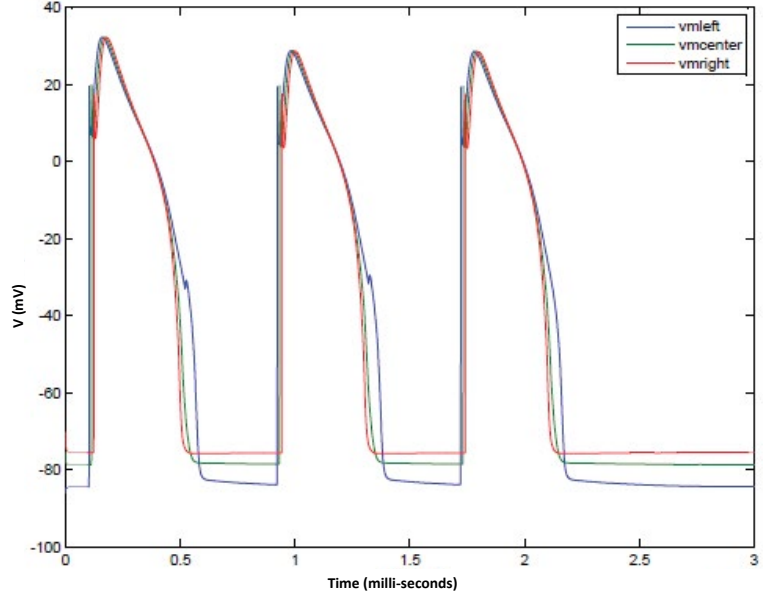

Figure 13: AP wave with left region $5.4 \mathrm{mM}$ and right at $8 \mathrm{mM}$. Time interval chosen, were: $100<t<101 ; 520<t<521 ; 920<t<921$; and $1720<t<7211$; the left but then failure on the right.

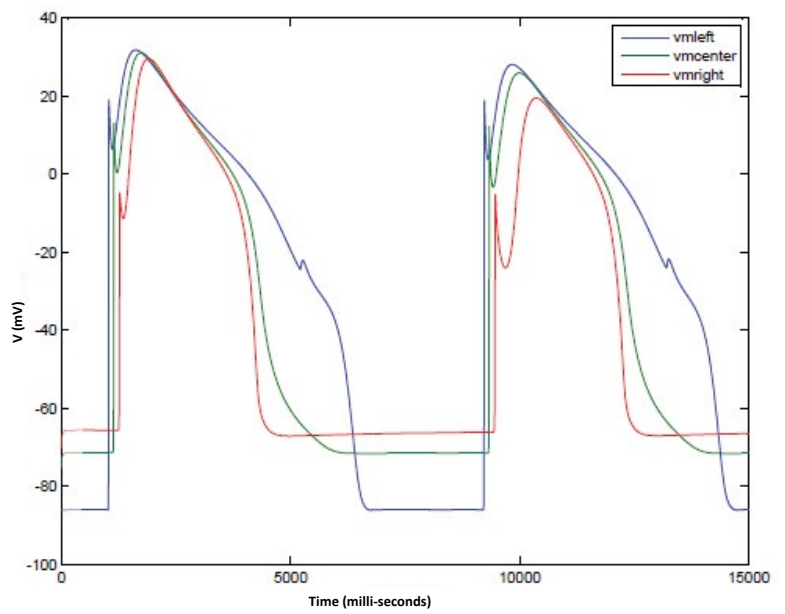

Figure 14: AP wave with left region $8 \mathrm{mM}$ and right at $12 \mathrm{mM}$ but for the same time intervals and stimulus voltage as in Figure 13.

a mathematical model, wherein we incorporated Luo Rudy's representation of the cell membrane current with a bidomain model of the cardaic tissue.

In addition to matching experimental results by Sidorov, reliability of our model is also demonstrated by reproduction results of previously reported models that predicted dependence of extracellular potassium on action potential duration.

Using our model, we examined the propagation of potassium from low to high concentration and found that $[K]_{0}$ is dependent on the action potential duration. Further research is necessary to refine our modelling approach by making it more inclusive of physiological parameters.

\section{Acknowledgments}

We thank Professor Bradley Roth (Oakland University) for his important suggestion and careful editing of the manuscript.

\section{References}

1. Roth BJ, Patel SG (2003) Effects of elevated extracellular potassium ion concentration on anodal excitation of cardiac tissue. J Cardiovasc Electrophysiol 14: $1351-1355$
2. Sidorov VY, Uzelac I, Wikswo JP (2011) Regional increase of extracellular potassium leads to electrical instability and reentry occurrence through the spatial heterogeneity of APD restitution. Am J Physoiol Heart Circ Physiol 301: $\mathrm{H} 209-\mathrm{H} 220$.

3. Hodgkin AL, Huxley AF (1952) A quantitative description of membrane current and its application to conduction and excitation in nerve. J Physiol 117: 500544.

4. Ebihara L, Johnson EA (1980) Fast sodium current in cardiac muscle. A quantitative description. Biophys J 32: 779-790.

5. Beeler GW, Reuter H (1977) Reconstruction of the action potential of ventricular myocardial fibres. J Physiol 268: 177-210.

6. Shibasaki T (1987) Conductance and kinetics of delayed rectifier potassium channels in nodal cells of the rabbit heart. J Physiol 387: 227-250.

7. Sakmann B, Trube G (1984) Conductance properties of single inwardly rectifying potassium channels in ventricular cells from guinea-pig heart. $J$ Physiol 347: 641-657.

8. Yue DT, Marban E (1988) A novel cardiac potassium channel that is active and conductive at depolarized potentials. Pflugers Arch 413: 127-133.

9. Muler AL, Markin VS (1977) [Electrical properties of anisotropic neuromuscular syncytia. I. Distribution of the electrotonic potential]. Biofizika 22: 307-312.

10. Miller WT, Geselowitz DB (1978) Simulation studies of the electrocardiogram. I. The normal heart. Circ Res 43: 301-315.

11. Tung L (1978) A bidomain model for describing ischemic myocardial dc potentials. PhD Dissertation, Massachusetts Institute of Technology, Cambridge, MA.

12. Plonsey R, Barr RC (1984) Current flow patterns in two-dimensional anisotropic bisyncytia with normal and extreme conductivities. Biophys J 45: 557-571.

13. Barr RC, Plonsey R (1984) Propagation of excitation in idealized anisotropic two-dimensional tissue. Biophys J 45: 1191-1202.

14. Roth BJ, Wikswo JP Jr (1986) A bidomain model for the extracellular potentia and magnetic field of cardiac tissue. IEEE Trans Biomed Eng 33: 467-469.

15. Roth BJ (1991) Action potential propagation in a thick strand of cardiac muscle. Circ Res 68: 162-173.

16. Sepulveda NG, Roth BJ, Wikswo JP Jr (1989) Current injection into a twodimensional anisotropic bidomain. Biophys J 55: 987-999.

17. Luo CH, Rudy Y (1991) A model of the ventricular cardiac action potential. Depolarization, repolarization, and their interaction. Circ Res 68: 1501-1526.

18. Luo CH, Rudy Y (1994) A dynamic model of the cardiac ventricular action potential. I. Simulations of ionic currents and concentration changes. Circ Res 74: 1071-1096.

19. Roth, BJ, Chen J (1999) Mechanism of anode break excitation in the heart: The relative influence of membrane and electrotonic factors. J Biol Systems 7: 541-552.

\section{Appendix}

Governing equations, parameters and their corresponding values for the Luo Rudy Model

$$
\begin{aligned}
& \frac{d V}{d t}=-\left(\frac{1}{C}\right)\left(I_{i}+I_{s t}\right) \\
& \frac{d y}{d t}=\frac{\left(y_{\infty}-y\right)}{\tau_{y}} \\
& \tau_{y}=\frac{1}{\left(\alpha_{y}+\beta_{y}\right)} \\
& y_{\infty}=\frac{\alpha_{y}}{\left(\alpha_{y}+\beta_{y}\right)} \\
& I_{N a}=23 m^{3} h j\left(V-E_{N a}\right)
\end{aligned}
$$

For $V \geq-40 m V$

$$
\alpha_{h}=\alpha_{j}=0.0, \beta_{h}=\frac{1}{\left(0.13\left\{1+\exp \left[\frac{V+10.66}{-11.1}\right]\right\}\right)}
$$


Citation: Dhungana S, Kar P (2015) Simulation of Propagation of Action Potentials in Cardiac Tissue with an Inhomogeneous Distribution of Extracellular Potassium. J Comput Sci Syst Biol 8:6 373-379. doi:10.4172/jcsb.1000212

$\beta_{j}=0.3 \cdot \exp \left(-2.535 \cdot 10^{-7} V\right) /\{1+\exp [-0.1(\mathrm{~V}+32)]\}$

For $\mathrm{V}<-40 \mathrm{mV}$

$\alpha_{h}=0.135 \cdot \exp \left[\frac{80+V}{-6.8}\right]$

$\beta_{h}=3.56 \exp (0.079)+3.1 . \exp (.35 \mathrm{~V})$

$\alpha_{j}=\left[-1.2714 .10^{5} \cdot \exp (0.2444 \mathrm{~V})-0.3474 .10^{-5} \cdot \exp (-0.04391 \mathrm{~V})\right]$

$(\mathrm{V}+37.78) /\{1+\exp [0.311 .(\mathrm{V}+79.23)]\}$

$\beta_{j}=\frac{.1212 \exp (-0.01052 \mathrm{~V})}{\{1+\exp [-0.1378(V+40.14)]\}}$

For all ranges of $\mathrm{V}$ :

$\alpha_{m}=\frac{.32(V+47.13)}{\{1-\exp [-0.1(V+47.13)]\}}$

$\beta_{m}=0.08 \exp \left(\frac{-V}{11}\right)$

Slow inward Current:

$I_{s i}=0.09 \mathrm{df}\left(V-E_{s i}\right)$

$E_{s i}=7.7-13.0287 \ln ([\mathrm{Ca}])$

$\alpha_{d}=\frac{.095 \exp [-0.01(V-5)]}{\{1+\exp [-0.072(V-5)]\}}$

$\beta_{d}=\frac{.07 \exp [-0.017(V+44)]}{\{1+\exp [0.05(V+44)]\}}$

$\alpha_{f}=\frac{.012 \exp [-0.008(V+28)]}{\{1+\exp [0.15(V+28)]\}}$

$\beta_{f}=\frac{.0065 \exp [-0.02(V+30)]}{\{1+\exp [-0.2(V+30)]\}}$

Calcium uptake: $\mathrm{d}\left([\mathrm{Ca}]_{\mathrm{i}} / \mathrm{dt}=-10^{-4} \cdot \mathrm{I}_{\mathrm{si}}+0.07\left(10^{-4}-[\mathrm{Ca}]_{\mathrm{i}}\right)\right.$

Expressions for outward currents are given below.

Time dependent Potassium Current:

$I_{K}=\overline{G_{K}} X X_{i}\left(V-E_{K}\right)$
$\overline{G_{K}}=0.282 \sqrt{\frac{[K]_{0}}{5.4}}$

$X_{i}=\frac{2.837 *\{\exp [0.04 *(V+77)]-1\}}{\{(V+77) * \exp [0.04(V+35)]\}}$ for $\mathrm{V}>-100 \mathrm{mV}$ and $\mathrm{X} i=1$ for $\mathrm{V} \leq-100 \mathrm{mV}$

$\alpha_{X}=\frac{.0005 \exp [0.083 *(V+50)]}{\{1+\exp [0.057(V+50)]\}}$

$\beta_{X}=\frac{.0013 \exp [-0.06(V+20)]}{\{1+\exp [0.04(V+20)]\}}$

Time- independent potassium current:

$I_{K 1}=\overline{G_{K 1}} K 1_{x}\left(V-E_{K 1}\right)$

$\overline{G_{K 1}}=0.6047 \sqrt{\frac{[K]_{0}}{5.4}}$

$\alpha_{K 1}=\frac{1.02}{\left\{1+\exp \left[0.02385\left(V-E_{K 1}-59.215\right)\right]\right\}}$

$\beta_{K 1}=\frac{\left\{0.49124 \exp \left[0.08032\left(V-E_{K 1}+5.476\right)\right]+\exp \left[0.06175\left(V-E_{K 1}-594.31\right)\right]\right\}}{\left\{1+\exp \left[-0.5143 *\left(V-E_{K 1}+4.753\right)\right]\right\}}$

Plateau Potassium current:

$I_{K \rho}=0.0183 K_{\rho}\left(V-E_{K \rho}\right)$

$E_{k p}=E_{k 1}$

$K_{p}=\frac{1 .}{\left\{1+\exp \left[\frac{7.488-V}{5.98}\right]\right\}}$

Background current:

$I_{b}=0.03921(V+59.87)$

Total time independent potassium current:

$I_{K 1(T)}=I_{K 1}+I_{K \rho}+I_{b}$ 